that Urasin is making steady progress in the previously reported investigations at the Engelhardt Observatory, Kazan, including SA at intermediate and high latitudes.

A few radial velocity measurements for stars in the SA should be mentioned. Griffin (06.112.011) has published pe determined radial velocities for more than 500 stars in the $+15^{\circ}$ SA-zone, including some high and intermediate areas. Bond et al. (06.112.014) have given radial velocities and spectral classifications for A-type stars near the SGP. It may also be mentioned that Berger et al. (06.112.004) propose that a $B$ star in the NGP cap with high radial velocity and at a distance of about $5 \mathrm{kpc}$ in $z$ may be ejected from disk to halo. Ardeberg's above mentioned spectrographic observations in the southern SA also include radial velocity determinations.

Haug reports that Schroeder of the Hamburg Observatory has made polarization measures at ESO for about 500 stars in the SGP cap $\left(b<-45^{\circ}\right)$. The data permit not only a discussion of the increase of polarization with distance from the Sun, but also a study of the differences in different directions. Ardeberg, also at ESO, has made polarization measurements for about 40 bright stars in SA 165, 188, and 205.

\title{
REFERENCES
}

Albers, H. 1972, Astrophys. J., 176, 623.

Ardeberg, A., Sarg, K., Wramdemark, S. $\quad 1972$, Astron. Astrophys. Suppl. Ser. (in press).

Crutcher, R. M. 1972, Ph. D. Thesis, Univ. California, Los Angeles, Calif.

Drilling, J. S. 1972, Astron. J., 77, 463.

Fujimoto, M., Kawabata, K., Sofue, Y. 1971, Prog. Theor. Phys. Suppl., 49, 181.

Graham, J. A., Miller, E. W. $\quad$ 1972, Bull. Am. Astron. Soc., 4, 312.

Hall, R. T. $\quad$ 1972, A Catalogue of Ten-Micron Celestial Objects, Aerospace Rept. No. TR-0172 (9260-01)-

17; The Aerospace Corporation, Los Angeles, Calif.

Hine, A. A. $\quad 1971$, A Catalogue of Nebulae in Carina, Steward Obs., Tucson, Arizona.

Hoffman, W. F., Frederick, C. L., Emery, R. J. $\quad$ 1971, Astrophys. J. Letters, 170, L89.

Hughes, E. E. 1972, Astron. J. (in press).

Iwanowska, W. 1972, Bull. Astron. Obs. Torún, No. 49.

Karlsson, B. $\quad$ 1972, Astron. Astrophys. Suppl. Ser., 7, 35.

Kerr, F. J., Knapp, G. R. $\quad$ 1972, Astron. J., 77, 573.

Knapp, G. R. $\quad 1972$, Ph. D. Thesis, Univ. Maryland, College Park, Md.

Kubinec, W. R. $\quad 1973$, Publ. Warner and Swasey Obs. (in press).

Kuznetsov, V. I. 1968, Voprosy Astrofiziki, Kiev: 'Naukova Dumka', p. 109.

Kuznetsov, V. I. $\quad 1972$ a, Astrometriya i Astrofiz., Kiev, No. 15.

Kuznetsov, V. I. $1972 b$, Abastumansk. Obs. Bull. (in press).

Lodén, L. O., Sundman, A. $\quad$ 1972, Astron. Astrophys., 20, 49.

Menon, T. K. 1971, in B. T. Lynds (ed.), Dark Nebulae, Globules and Protostars, Univ. Arizona Press, Tucson, Arizona, p. 67.

Nordstrom, B., Sundman, A. 1972, IAU Symp. No. 50 (to be published).

Pesch, P. 1972, Astrophys. J., 177, 519.

Philip, A. G. D. 1972a, ESO. Conf. The Role of Schmidt Telescopes in Astronomy, Hamburg, (to be published).

Philip, A. G. D. 1972b, IAU Symp. No. 52 (to be published).

Philip, A. G. D., Stock, J. 1972 , Bol. Obs. Tonantzintla Tacubaya (to be published).

Priser, J. B. 1972, Publ. U.S. Naval Obs. (to be published).

Simonson, S. C., Sancisi, R. $\quad$ 1972, Astron. Astrophys. Suppl. Ser. (in press).

Smith, B. M. 1972, Catalogue of Nebulae in Centaurus, Crux, Circinus and Norma, Steward Obs., Tucson, Arizona (in press).

Stegman, J. E., FitzGerald, M. P. 1972, J. Roy. Astron. Soc. Canada (in press).

Sturch, C. R., Sharpless, S. $\quad 1972$, Astron. J., 77, 669.

Wilson, W. J. F., FitzGerald, M. P. $\quad$ 1972, J. Roy. Astron. Soc. Canada, 66, 254.

Wooden, W. H. II. 1971, Publ. Warner and Swasey Obs., 1, No. 2.

\section{OVERALL STRUCTURE OF THE GALAXY}

Sections II and III of this report have dealt with details of the galactic structure within reach of 
most present day optical observational techniques. In the present section the larger-scale features of the Galaxy will be discussed, revealed to a large extent by radio astronomical data coupled with the optical.

\section{A. Spiral structure}

Two small conferences held to discuss problems of galactic spiral structure have been reviewed by Simonson (04.155.012) and Bok et al. (1972). The main observational data that can be used for mapping the spiral structure of the whole Galaxy are those obtained in the $21-\mathrm{cm}$ hydrogen line. Other types of observation can be used for comparison with a $21-\mathrm{cm}$ pattern, but so far they cannot lead to an independently-derived pattern for the whole Galaxy. The most recent review of the problems involved in 21-cm spiral structure studies is by Burton (07.155.061). There seems to be reasonable agreement that optical spiral arm tracers such as OB stars, $\mathrm{H}$ II regions, long-period cepheids and the like must be observed at larger distances in order to reinforce the radio observations at distances of the order of 5 to $8 \mathrm{kpc}$. And this depends on observational power to reach OB stars, for example, to $m_{\mathrm{pg}} \sim 15-16$. Bok and his colleagues have demonstrated that this can be done (see Section III). Two 21 -cm diagrams for the $\mathrm{H}$ I distribution over the galactic plane have been presented by Kerr $(03.155 .026)$ and by Weaver $(03.155 .028 ; 1972)$. These are based on the assumption that peaks in the line profiles are due primarily to density variations in the $\mathrm{H}$ I gas. Each of these patterns has an average pitch angle of about $7^{\circ}$ for the spiral arms.

It has long been recognized that the derived spiral patterns are very dependent on assumptions about the galactic rotation curve and additional non-circular components of motion. The densitywave theory provides a suitable framework for introducing studies of these effects. Burton $(05.155 .001 ; 1972)$ in particular has emphasized that the velocity field of the Galaxy and the $\mathrm{H} \mathrm{I}_{\mathrm{I}}$ density distribution must be studied simultaneously. He has used the density-wave theory to produce a map of the Sagittarius spiral arm. Yuan and Liebovitch (07.155.059) have derived a regular 'grand design' which agrees with the observational data on a large scale.

While detailed studies have been reported for smaller regions, nobody has yet derived a detailed map of the hydrogen density distribution for the whole Galaxy, allowing both velocity and density variations to be responsible for the profile shapes. Tuve and Lundsager (1972) have in fact gone so far as to suggest that the streaming motions and other velocity variations will effectively mask whatever density concentrations may exist.

An alternative view of spiral structure as a magnetically-generated phenomenon has been proposed by Piddington (1972) whose hydromagnetic theory of twin spiral arms is based on gas motions mainly perpendicular to the disk caused by a field whose tilt varies sinusoidally with rotation. Star formation is controlled by gas streaming obliquely along field lines.

Radio and optical investigations have led to different spiral patterns in some regions. Kerr (03.155.026) and Weaver (1972) regard the Carina and Sagittarius arms as separate features and Kerr and Kerr (04.155.002) have shown that the longitude distribution of the flux in the radio continuum and in recombination-line sources show the existence of a gap between these two arms. On the other hand, Georgelin and Georgelin $(03.155 .055 ; 05.155 .027)$ consider that the Sagittarius and Carina arms are joined together as a major feature through the Centaurus region. Their conclusions are drawn from kinematic studies of many Hil regions and spectrophotometric measurements of the exciting stars. Humphreys (1972) draws the Sagittarius and Carina arms as separate features, but with a possible cross-connection between them. The structure in Carina has been studied in detail by Garzoli (04.155.003). Whether the local feature, with the Sun on the edge nearest the galactic center is a bonafide spiral arm segment or an interarm link remains to be resolved.

Bok (05.155.009) and Miller (07.113.016) have summarized current knowledge concerning the Carina-Centaurus spiral complex; viz. (a) it is confined between $l=280^{\circ}$ and $300^{\circ}$ with a relatively sharp outer boundary (away from the galactic center) beginning at $2 \mathrm{kpc}$ and extending to at least $8 \mathrm{kpc}$ from the Sun; (b) there is a central core of bright HiI regions, OB stars, long-period cepheids, WR stars, young clusters and associations in the range $l=287^{\circ}$ to $295^{\circ}$; (c) there is a broad band of neutral hydrogen 0.7 to $1.0 \mathrm{kpc}$ wide extending over much of the $l, b$ range in which the central 
core is embedded; (d) along the outer edge $\left(l=280^{\circ}-283^{\circ}\right)$ the interstellar absorption increases rapidly with distance from the Sun to $A_{v} \sim 3-4$ mag at distances perhaps as small as $4 \mathrm{kpc}$, while along the inner edge $\left(l=295^{\circ}\right)$ the values of $A_{\mathrm{v}}$ are much smaller within at least $4 \mathrm{kpc}$.

The extensive research by Humphreys (1972) indicates good agreement between the radial velocities of the supergiant stars and the densest features of $\mathrm{HI}$ gas and of the HII regions in the Carina spiral feature. Miss Humphreys is now beginning a study of the Centaurus-Norma region $\left(305^{\circ}<l<\right.$ $<335^{\circ}$ ) to examine the possibility of a link between the Carina and Sagittarius arms.

In his study of $O B$ stars from relatively high dispersion spectra Walborn (06.114.101) has confirmed evidence for the local spiral feature of the Galaxy extending from the Sun to a distance of $1.2 \mathrm{kpc}$ at $l=135^{\circ}$. There are very few $\mathrm{OB}$ stars in the gap between the local and Perseus spiral features, followed by a well defined concentration in the Perseus arm at $2.5 \mathrm{kpc}$. A comparable discussion by Crampton (07.115.003) of 49 high-luminosity stars in the Perseus arm, selected on the basis of their double Ca II lines, indicates that the spiral feature between $l=120^{\circ}$ and $140^{\circ}$ has considerable width, extending from 1.8 to $3.0 \mathrm{kpc}$ from the Sun.

Minn and Greenberg (1972) have studied the kinematical and the spatial coincidence of the optical and radio spiral features of the Galaxy. Among other things their analysis indicates that if the discontinuity at $l=140^{\circ}$ in the Perseus spiral arm is due to a heavy dust complex marking a continuation of the spiral arm the pitch angle of some $20^{\circ}-25^{\circ}$ exhibited by the optical tracers is much reduced and becomes comparable with the $6^{\circ}-7^{\circ}$ pitch angle exhibited by the $\mathrm{HI}$ gas distribution.

The relation between $\mathrm{H}_{2} \mathrm{CO}$ absorption clouds and spiral structure has been discussed by Whiteoak and Gardner (03.131.004) and Wilson (1972) and a similar discussion for recombinationline sources has been given by Wilson et al. (03.131.095). Recombination-line emission at a very low level was detected at points along the galactic ridge between the known discrete sources by Gottesman and Gordon (04.131.114) and by Jackson and Kerr (06.131.014). This emission appears to arise in distributed ionization in spiral arms, an interpretation supported by the general agreement in shape with the corresponding $21-\mathrm{cm}$ line profiles from neutral hydrogen. Additional observations have been reported by Gordon and Cato (1972), and by Davies et al. (1972). In principle, these investigations provide another way to examine the spiral structure, but the radiation is so weak that it has only been detected from a small number of points so far. The emission appears to be concentrated to the region $4-8 \mathrm{kpc}$ from the galactic center, where the giant $\mathrm{HII}$ regions are also found.

A flurry of activity on problems relating to the stellar rings introduced by Isserstedt and SchmidtKaler in 1967 as possible spiral arm tracers has taken place during the past three years. A general discussion of these rings, their relation to star formation and to problems of galactic spiral structure has been given by Schmidt-Kaler (1970) at the 14th Herstmonceux Conference on The Distances and Sizes of Cosmic Objects. If they are physically related groups of young stars, and if the minor diameters of the elliptical rings are uniformly about $7 \mathrm{pc}$ then Isserstedt (04.155.009) has found the distribution on the galactic plane agrees well with other optical tracers. All of this hinges on the reality of the rings as physical entities. Evidence pro and con has been submitted by Isserstedt and Schmidt-Kaler (04.152.002; 07.152.008), Isserstedt (02.152.004; 04.114.017), Haug and Kohoutek (05.152.001), Schild and Cowley (06.152.001), Kolesnik (07.152.001), Crampton and Byl (05.152.012) and Prochaska $(05.152 .009 ; 07.152 .011)$. Much remains to be done to establish the reality of the groups on the extensive scale now assumed.

\section{B. The galactic disk}

The contributions of optical astronomy to the overall structure of the Galaxy near the galactic plane have been covered for the most part in Section III. A few of somewhat broader scope will be mentioned here. We consider primarily the properties of radio emission and absorption which refer to the disk in general, with no special connection with spiral structure.

The ratio of blue to red supergiant stars $[N(B) / N(R)]$ as a function of distance from the galactic 
center has been studied by Hartwick (04.155.026) with data published by Humphreys (03.155.054). There appears to be a marked decrease in this ratio from 12 at $R=7.5 \mathrm{kpc}$ to 2 at $R=12.2 \mathrm{kpc}$. Whether this implies a change in average chemical content of the stars as a function of position relative to the galactic center remains to be determined. Some evidence for such a gradient comes from a photometric classification of $\mathrm{G}$ and $\mathrm{K}$ stars by Grenon at Geneva, who finds that the metalrich and especially the super-metal-rich stars now observable near the Sun appear to be disk members formed closest to the galactic center. The identity of age and kinematic properties between giants and dwarfs indicates that supermetallicity results from primordial chemical composition rather than from stellar evolutionary effects.

The relationship between the distributions of planetary nebulae and galactic clusters has been investigated by Akhundova and Seidov (04.133.002) for $|b| \leqslant 10^{\circ}$. As expected the planetary nebulae do not tend toward the spiral features of the Galaxy as do the young clusters, but are concentrated toward the galactic center.

Wilson and Barrett $(05.114 .085 ; 07.114 .048)$ have observed 403 infrared stars and 19 other objects to see whether $\mathrm{OH}(1612 \mathrm{MHz})$ was present. In 23 of these $\mathrm{OH}$ emission was detected, the majority being Mira variables in which the infrared radiation is due most probably to circumstellar shells. The sources in which $\mathrm{OH}$ has been detected appear to be concentrated toward the galactic plane and toward the galactic center. Their probable distances are less $4 \mathrm{kpc}$.

Goldstein (07.155.035) has estimated the total amount of neutral hydrogen in the Galaxy by a new method. His value is $2.3-2.9 \times 10^{9} M_{\odot}$, in good agreement with determinations based on integration over the whole distribution pattern. Kellman (1972a, b) has considered the equilibrium configuration of the gaseous disk, and has compared his computed values for the half-thickness in the range $R=4-14 \mathrm{kpc}$ with earlier observations.

The large-scale properties of galactic radio sources have been discussed by Shaver and Goss (04.141.104). Parrish (07.141.124) has derived the brightness temperature of the continuum radiation from the galactic disk by subtracting out the contributions from thermal radio sources, as derived from spectral observations at seven frequencies between 50 and $320 \mathrm{MHz}$. Higgs $(06.157 .007)$ has estimated the rms confusion levels in the galactic plane, on the basis of a model distribution of thermal radio sources in the Galaxy, and finds that thermal emission can account for most of the galactic background at frequencies above $3 \mathrm{GHz}$.

Ariskin (05.155.022) has shown that a spatial relationship exists between a number of the largescale regions of ionized hydrogen responsible for the thermal component of the radio background and discrete recombination line sources. Altenhoff and Wilson (03.141.200) have considered the apparent asymmetry between the northern and the southern Milky Way in the relative numbers of thermal and nonthermal sources. They suggest that the reported differences may be largely due to the use of different radio telescopes in the surveys in the two hemispheres.

The strongest radio continuum sources, except at the shortest wave-length, are the supernova remnants. Over 120 have now been catalogued in the Galaxy by Milne (03.125.016), Downes (05.125.018) and Ilovaisky and Lequeux (07.125.012). The whole subject has been reviewed by Woltjer (1972). The galactic distribution of SNRs exhibits a reasonable degree of clustering along spiral arms, indicating that they are extreme Population I objects. The $z$-distribution is quite similar to that for the Hr gas, with a strong concentration toward the galactic plane.

\section{The galactic nucleus}

The galactic center region has been studied by many investigators in the radio continuum, the 21-cm hydrogen line, molecular lines, radio recombination lines, and in the far infra-red. In the continuum, the small-diameter source Sgr A, which is presumed to be at the very center, has been observed with high resolution by Sandqvist (1972), Krishna et al. (1972) and Ekers and Lynden-Bell (06.155.034). All have found additional compact components. Dworetsky et al. (02.141.164) and Lipovka (05.141.086) have observed the source at short wavelengths where thermal emission dominates, and find that the apparent size of the source decreases with increasing radio frequency. At 
the low frequency of $80 \mathrm{MHz}$ Dulk (04.141.160) finds no evidence for a small-diameter source with a flux density over $5 \mathrm{f}$.u. in observations with a $3 \cdot 8^{\prime}$ beam.

Lynden-Bell and Rees (05.155.040) have discussed the properties of quasars as proto-black-holes in the middle of galaxies, and have listed critical observations which could establish the existence of a large central mass in our Galaxy small enough to be associated with a black hole.

In the 21-cm HI line Simonson (05.155.019) has identified neutral hydrogen concentrations with high positive velocity apparently belonging to the inner region of the Galaxy. Masses of $10^{5}-10^{6} M_{\odot}$ and distances from the galactic plane of $200-1300 \mathrm{pc}$ are found.

Van der Kruit $(03.155 .017 ; 06.155 .002)$ has found features that suggest the ejection of clouds from the nucleus in two roughly opposite directions at a large angle to the plane. He has also proposed a model in which the expanding features in the galactic plane were formed by material ejected originally out of the plane, which has now fallen back into the quiescent gas layer.

Extensive occultation observations in the $\mathrm{OH}$ and formaldehyde lines [Sandqvist (06.155.050; 1972)] have shown considerable fine structure in the absorbing molecular clouds in front of Sgr A. In particular, a cloud with a central velocity of $+40 \mathrm{~km} \mathrm{~s}^{-1}$ apparently close to the center, has a diameter of $8 \mathrm{pc}$ and is rotating with speeds up to $25 \mathrm{~km} \mathrm{~s}^{-1}$ in the same direction as that of galactic rotation. This particular cloud, together with molecular clouds in Sgr B2 at a projected distance of $100 \mathrm{pc}$ from the center, are the principal places in the Galaxy where new molecules have been discovered and their astrophysical properties studied. [See Commission 34 and 40 reports for details].

The distribution of $1667-\mathrm{MHz} \mathrm{OH}$ absorption over several square degrees near the galactic center has been extensively studied by Robinson and McGee (03.131.124), McGee et al. (04.131.117) and McGee (04.131.046 and .124), who have recognized 63 hydroxyl clouds and listed their properties. They also describe the motions of $\mathrm{OH}$ near the galactic nucleus, with apparent differences from those deduced from hydrogen-line measurements. Similarly, the formaldehyde absorption over the central region has been mapped by Gardner and Whiteoak $(03.131 .029 ; 07.155 .030)$ and Scoville et al. (07.131.029). Further results on motions in the nucleus will be discussed in Section V.

The center of the Galaxy has been observed in the far infrared (40-350 $\mu$ ) by Aumann and Low (03.155.009). They describe a source with a diameter $<3^{\prime}$, within $6^{\prime}$ of the position of Sgr A, and consider that size and luminosity considerations strongly favor a nonthermal model of the galactic nucleus with multiple sources. Hoffmann et al. (05.141.061) have published a detailed map over an emitting region $2^{\circ} \times 3.6$, in the spectral interval $75-125 \mu$ with a resolution of $6^{\prime}$. Five individual peaks were observed. These authors favor a thermal dust-emission model with temperatures of $20-50 \mathrm{~K}$. Emission from dust is also preferred by Okuda and Wickramasinghe (03.155.020), Lequeux (03.155.007) and Pottasch (05.155.039). The strong emission implies a large total mass of dust, so that the interstellar mass in the central region may be larger than that indicated by $21-\mathrm{cm}$ observations. Sanders and Lowinger (07.155.041) have computed a mass model for the nucleus, assuming that the distribution of the infrared emission follows that of the stellar mass.

Houck et al. (06.155.032) have observed the galactic center region via an Aerobee 170 rocket equipped with 4-color infrared detectors extending over the range 5 to $115 \mu$. They detected 5 sources of which one, the galactic nucleus, was previously known. The galactic nucleus has been studied at $\lambda=11800 \AA$ and $10600 \AA$, where the airglow has minimal effect, by Spinrad et al. $(05.155 .020)$. They find that the near-infrared spectrum of the galactic nucleus is similar to that of M31 and the visual interstellar absorption between the Sun and the galactic center, $A_{v}$, is about 29 mag.

A summary, or review, of the status of infrared astronomical research by Hyland (06.114.062) contains, in addition to stellar and interstellar data, comments about the nucleus of the Galaxy.

Bradt et al. (05.142.005) have surveyed the galactic center region for X-ray sources and have discovered 11 between $l=335^{\circ}$ and $20^{\circ}$ (through $0^{\circ}$ ) and $-10^{\circ}<b<+10^{\circ}$. Their survey covered the 1-10 keV X-ray region. The sources appear to be small, show no evidence of flux at radio wavelengths, no evidence of variability, and no positional correlation with other known sources of energy. 


\section{The galactic halo}

Section IIIC of this report treats the progress made by optical techniques in problems of galactic structure at $|b|>20^{\circ}$. In the present section larger-scale phenomena revealed by radio observational techniques are of primary concern. Gehrz and Woolf (05.064.031) have deduced from infrared photometry the rates of mass loss from $M$ stars, principally Mira variables. Then from prior data on the volume density of such stars they have calculated the rate of mass returned onto the galactic plane as of the order of $6 \times 10^{-10} M_{\odot} \mathrm{yr}^{-1} \mathrm{pc}^{-2}$. When augmented by other halo and disk objects the total mass loss by stars is $7 \times 10^{-10} M_{\odot}$.

Several authors have considered the possibility of a radio halo. Razin (05.157.001) discussed Mathewson's polarization data, as well as the distribution of radio emission over the sky, and concluded that the local spurs and other features confuse the halo question considerably, but at least a strong halo is ruled out; a weak halo could exist. Clark et al. (04.157.011) from a study of low-frequency satellite and ground based observations from 0.5 to $100 \mathrm{MHz}$ conclude that any halo contribution is below $15 \%$ of the overall brightness at $100 \mathrm{MHz}$, and the halo volume emissivity does not exceed $1 \%$ of the galactic disk emissivity. On the other hand, Abramjan et al. (03.157.022) derive models from observations in the range $96-150 \mathrm{MHz}$ which require a relatively large radio halo in addition to the disk component.

The high-velocity gas will be considered in Section V B. There is some controversy over its distance and location in relation to the galactic structure.

\section{REFERENCES}

Bok, B. J., Cordwell, C. S., Humphreys, R. M. (eds.) $\quad$ 1972, Problems of Galactic Spiral Structure, Steward Obs. Tucson, Arizona.

Burton, W. B. 1972, in G. L. Verschuur, K. I. Kellerman (eds.) Galactic and Extragalactic Astronomy, Springer-Verlag, New York (in press).

Davies, R. D., Matthews, H. E., Pedlar, A. $\quad$ 1972, Nature Phys. Sci., 238, 101.

Gordon, M. A., Cato, T. 1972, Astrophys. J., 176, 587.

Humphreys, R. M. 1972, Astron. Astrophys., 20, 29.

Kellman, S. A. 1972a, Astrophys. J., 175, 353.

Kellman, S. A. $\quad 1972$ b, Astrophys. J., 175, 363.

Krishna, G., Swarup, G., Sarma, N. V. G., Joshi, M. N. $\quad$ 1972, Nature, 239, 91.

Minn, Y.-K., Greenberg, J. M. 1972, Astron. Astrophys. (in press).

Piddington, J. H. 1972, Cosmic Electrodynamics, 3, 129.

Sandqvist, A. 1972, Astron. Astrophys. Suppl. Ser. (in press).

Schmidt-Kaler, T. 1970, Observatory, 90, 213.

Tuve, M. A., Lundsager, S. 1972, Astron. J., 77, 652.

Weaver, H. F. 1972, in B. J. Bok, C. S. Cordwell, R. M. Humphreys (eds.), Problems of Galactic Spiral Structure, Steward Obs. Tucson, Arizona, p. 28.

Wilson, T. L. 1972, Astron. Astrophys. 19, 354.

Woltjer, L. $\quad$ 1972, Ann. Rev. Astron. Astrophys., 10, 129.

\section{KINEMATICS}

\section{A. Stars}

\section{Galactic Rotation}

A survey of stellar kinematics with its historical background and relation to stellar evolution has been given by Eggen (05.115.017). A comprehensive investigation of the structure and evolution of the Galaxy, involving radial velocity measurements in the polar caps for the determination of the $z$-attraction and detection of the tilt of the velocity ellipsoid outside the galactic plane has been proposed by Einasto and Kuzmin (04.155.021). Einasto (1971) has discussed available kinematical characteristics of subsystems of stars in the Galaxy, has derived mean values for the principal populations and has estimated the age of populations. 